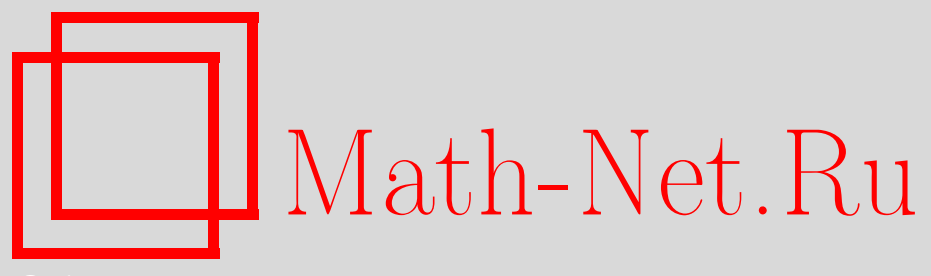

М. Асорей, Дж. Сколаричи, Л. Соломбрино, Комплексные проекции вполне положительных кватернионных отображений, ТМФ, 2007, том 151, номер 3, 360-370

DOI: https://doi.org/10.4213/tmf6051

Использование Общероссийского математического портала Math-Net.Ru подразумевает, что вы прочитали и согласны с пользовательским соглашением http://www . mathnet.ru/rus/agreement

Параметры загрузки:

IP: 35.174 .16 .151

26 апреля 2023 г., 11:46:27

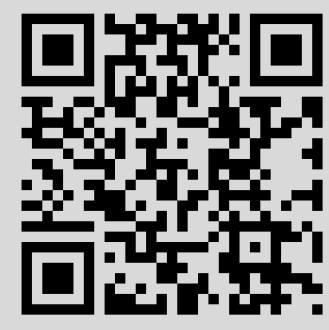




\title{
КОМПЛЕКСНЫЕ ПРОЕКЦИИ ВПОЛНЕ ПОЛОЖКТЕЛЬНЫХ КВАТЕРНИОННЫХ ОТОБРАЖЕНИЙ
}

\begin{abstract}
Показано, что комплексная проекция вполне положительного кватернионного отображения кватернионных матриц плотности является положительным отображением в пространстве комплексных матриц плотности. Дано краткое описание некоторых свойств таких проекций. В качестве примера исследована комплексная проекция однопараметрической кватернионной унитарной динамики квантовой системы со спином $1 / 2$.
\end{abstract}

Ключевые слова: открытые квантовые системы, положительные отображения, кватернионы.

\section{1. ВВЕДЕНИЕ}

Редукция волновых пакетов в квантовой механике представляет собой нелинейную процедуру, нелинейное обобщение теории было давно предложено Вайнбергом, который также предложил некоторые точные тесты для нелинейных поправок [1]. С другой точки зрения редукция волновых пакетов допускает описание в терминах открытых квантовых систем посредством положительных отображений в пространстве матриц плотности. В противоположность унитарной динамике, отображения, ассоциированные с редукцией волновых пакетов, переводят чистые состояния в смешанные, увеличивая таким образом их энтропию фон Неймана.

Данная работа посвящена анализу некоторых следствий кватернионной квантовой механики, значимых для теории квантовых измерений. Действительно, хотя связь вполне положительных отображений и составных состояний хорошо изучена [2], физическая интерпретация отображений, которые являются положительными, но не вполне положительными, по-прежнему представляет собой предмет исследования [3]. Существуют наблюдения, которые наводят на мысль, что кватернионная квантовая механика может оказаться полезной для классификации положительных отображений в комплексной квантовой механике. Мы полагаем, в

*Departamento de Física Teórica, Universidad de Zaragoza, 50009 Zaragoza, Spain. E-mail: asorey@saturno.unizar.es

${ }^{\dagger}$ Dipartimento di Fisica dell'Universitá di Lecce, INFN, Sezione di Lecce, I-73100 Lecce, Italy. E-mail: scolarici@le.infn.it, solombrino@le.infn.it 
частности, что некоторые полезные указания в этом направлении можно извлечь из недавно полученных результатов по кватернионным отображениям и их комплексным проекциям [4]-[6]. Цель настоящей работы - продолжить наши исследования комплексных проекций вполне положительных кватернионных отображений и охарактеризовать их подходящим (хотя и не исчерпывающим) образом.

Работа организована следующим образом. В разделе 2 кратко излагается формализм матриц плотности в кватернионной квантовой механике и вводится понятие энтропии фон Неймана для кватернионных состояний. В разделе 3 рассматриваются вполне положительные кватернионные отображения и их комплексные проекции. В разделе 4 изучается подмножество вполне положительных кватернионных отображений, а именно унитарные кватернионные отображения, и приводятся некоторые результаты относительно их комплексных проекций. Наконец, в разделе 5 общие результаты предыдущих разделов применяются к двухуровневой (не зависящей от времени) кватернионной квантовой системе. В разделе 6 собраны некоторые заключительные замечания.

\section{2. МАТРИЦА ПЛОТНОСТИ И ЭНТРОПИЯ ФОН НЕЙМАНА В КВАТЕРНИОННОЙ КВАНТОВОЙ МЕХАНИКЕ}

Напомним некоторые основные обозначения. Вещественный кватернион обычно записывается в виде

$$
q=q_{0}+q_{1} i+q_{2} j+q_{3} k,
$$

где $q_{l} \in \mathbb{R}, l=0,1,2,3, i^{2}=j^{2}=k^{2}=-1, i j=-j i=k$.

Кватернионное кососимметричное поле $\mathbb{Q}$ представляет собой алгебру ранга 4 над полем $\mathbb{R}$, которая является некоммутативной и снабжена инволютивным антиавтоморфизмом (операцией сопряжения), так что

$$
q \rightarrow \bar{q}=q_{0}-q_{1} i-q_{2} j-q_{3} k
$$

Каждому линейному оператору в правом $n$-мерном векторном пространстве $\mathbb{Q}^{n}$ над $\mathbb{Q}$ обычным образом сопоставляется $(n \times n)$-матрица, действующая слева. Кроме того, по аналогии со случаем векторных пространств над полем $\mathbb{C}$ можно ввести понятия унитарности, эрмитовости и т.д.

Матрица плотности $\rho_{\psi}$, соответствующая чистому состоянию $|\psi\rangle$, которое принадлежит кватернионному $n$-мерному правому гильбертову пространству $\mathbb{Q}^{n}$, определяется следующим образом [7]:

$$
\rho_{\psi}=|\psi\rangle\langle\psi|
$$

она одинакова для всех нормированных представителей луча. По определению матрицы плотности $\rho_{\psi}$, соответствующие чистым состояниям, представляют собой положительно-определенные кватернионные эрмитовы операторы ранга 1 с единичным следом на пространстве $\mathbb{Q}^{n}$. По аналогии с обычной (комплексной) квантовой механикой можно ввести понятие кватернионных смешанных состояний, которые 
определяются положительными кватернионными эрмитовыми операторами $\rho$ с единичным следом и рангом, большим единицы, на пространстве $\mathbb{Q}^{n}$. Положительность означает, что для любого чистого состояния $|\psi\rangle \in \mathbb{Q}^{n}$

$$
\langle\rho\rangle_{\psi}=\langle\psi|\rho| \psi\rangle=\operatorname{Re} \operatorname{tr}(\rho|\psi\rangle\langle\psi|)=\operatorname{Re} \operatorname{tr}\left(\rho \rho_{\psi}\right) \geqslant 0,
$$

где $\operatorname{Re} \operatorname{tr} C=\operatorname{Re}\left(\sum_{r} C_{r r}\right)=\sum_{r} \operatorname{Re} C_{r r}$ обозначает вещественную часть следа оператора $C$ (вещественный след обладает циклическим свойством $\operatorname{Re} \operatorname{tr} A B=\operatorname{Re} \operatorname{tr} B A$ [8]). Обратим внимание, что здесь и далее мы не требуем выполнения в формуле (2) строгого неравенства.

Математическое ожидание (среднее значение) кватернионного эрмитова оператора $A$ в состоянии $|\psi\rangle$ может быть выражено в терминах матрицы плотности $\rho_{\psi}$ следующим образом [7]:

$$
\langle A\rangle_{\psi}=\langle\psi|A| \psi\rangle=\operatorname{Re} \operatorname{tr}(A|\psi\rangle\langle\psi|)=\operatorname{Re} \operatorname{tr}\left(A \rho_{\psi}\right) .
$$

Полагая $A=A_{\alpha}+j A_{\beta}$ и $\rho=\rho_{\alpha}+j \rho_{\beta}$, где $A_{\alpha}, A_{\beta}, \rho_{\alpha}$ и $\rho_{\beta}$ - комплексные матрицы, легко видеть, что среднее значение $\langle A\rangle_{\psi}$ может зависеть от $A_{\beta}$ или $\rho_{\beta}$, только если как $A_{\beta}$, так и $\rho_{\beta}$ отличны от нуля, т.е. только если и наблюдаемая, и состояние представлены подлинными кватернионными матрицами. Действительно,

$$
\langle A\rangle_{\rho}=\operatorname{Re} \operatorname{tr}(A \rho)=\operatorname{Re} \operatorname{tr}\left(A_{\alpha} \rho_{\alpha}-A_{\beta}^{*} \rho_{\beta}\right),
$$

где * обозначает комплексное сопряжение.

Далее, если наблюдаемая $O$ описывается чисто комплексной эрмитовой матрицей, то ее среднее значение не зависит от кватернионной части $j \rho_{\beta}$ состояния $\rho=\rho_{\alpha}+j \rho_{\beta}$. Более того, среднее значение, предсказываемое комплексной квантовой механикой, для состояния $\rho_{\alpha}$ совпадает со средним значением, предсказываемым кватернионной квантовой механикой, для состояния $\rho=\rho_{\alpha}+j \rho_{\beta}$, поскольку

$$
\operatorname{tr}\left(O \rho_{\alpha}\right)=\operatorname{Re} \operatorname{tr}\left(O \rho_{\alpha}\right)=\operatorname{Re} \operatorname{tr}(O \rho)
$$

Как и в комплексной квантовой механике, можно ввести энтропию фон Неймана, соответствующую кватернионному квантовому состоянию $\rho$, которое описывает квантовую систему $S$ :

$$
S(\rho):=-\operatorname{tr}(\rho \ln \rho) .
$$

Энтропия фон Неймана, как и в обычной комплексной теории, представляет собой количественную меру неопределенности фактического состояния системы $S$. Действительно, эрмитовость оператора $\rho$ (и, следовательно, $\ln \rho$ ) влечет эрмитовость оператора $\rho \ln \rho$ (поскольку $\rho$ и $\ln \rho$ коммутируют), и мы имеем

$$
S(\rho)=-\operatorname{tr}(\rho \ln \rho)=-\operatorname{Re} \operatorname{tr}(\rho \ln \rho)=-\operatorname{Re} \operatorname{tr}\left(U \rho U^{\dagger} U(\ln \rho) U^{\dagger}\right)=-\sum_{s=1}^{n} \lambda_{s} \ln \lambda_{s},
$$

где $U$ обозначает кватернионное унитарное преобразование, диагонализирующее одновременно $\rho$ и $\ln \rho$, а $\lambda_{s}$ - вещественные собственные значения оператора $\rho$.

Оказывается, что $S(\rho)=0$ тогда и только тогда, когда $\rho^{2}=\rho$, в противном случае $0<S(\rho)<\ln n$. 


\section{3. ВПОЛНЕ ПОЛОЖИТЕЛЬНЫЕ КВАТЕРНИОННЫЕ ОТОБРАЖЕНИЯ И ИХ КОМПЛЕКСНЫЕ ПРОЕКЦИИ}

Обозначим через $M_{n}(\mathbb{Q})$ пространство кватернионных $(n \times n)$-матриц и через $H_{n}(\mathbb{Q})$ пространство кватернионных эрмитовых $(n \times n)$-матриц $\rho=\rho_{\alpha}+j \rho_{\beta}$. По аналогии с вполне положительными комплексными отображениями вида КраусаСтайнспринга [9], [10] примем следующее определение [4].

ОПРедЕЛЕНИЕ. Отображение $\Phi: H_{n}(\mathbb{Q}) \rightarrow H_{n}(\mathbb{Q})$ называется вполне положительным, если оно может быть записано в виде

$$
\Phi(\rho)=\sum_{s} U_{s} \rho U_{s}^{\dagger}
$$

где $U_{s} \in M_{n}(\mathbb{Q})$.

Следующие предложения полезны для понимания некоторых свойств отображений (7).

ПрЕДЛОЖЕНИЕ 1. Пусть $\rho$ - некоторая положительно-определенная кватернионная эрмитова матрица. Тогда вещественная линейная комбинация

$$
\sum_{s} \lambda_{s} V_{s} \rho V_{s}^{\dagger}, \quad \lambda_{s}>0
$$

где $V_{s}=\left(V_{\alpha}+j V_{\beta}\right)_{s} \in M_{n}(\mathbb{Q})$, является положительно-определенной кватернионной эрмитовой матрицей.

ДокАЗАТЕЛьСтво. Используя изоморфизм между кватернионными матрицами и их комплексными симплектическими разложениями, можно переписать эрмитову форму (8) в $2 n$-мерном комплексном пространстве следующим образом:

$$
\sum_{s} \lambda_{s}\left(\begin{array}{cc}
V_{\alpha} & V_{\beta} \\
-V_{\beta}^{*} & V_{\alpha}^{*}
\end{array}\right)_{s}\left(\begin{array}{cc}
\rho_{\alpha} & \rho_{\beta} \\
-\rho_{\beta}^{*} & \rho_{\alpha}^{*}
\end{array}\right)\left(\begin{array}{cc}
V_{\alpha} & V_{\beta} \\
-V_{\beta}^{*} & V_{\alpha}^{*}
\end{array}\right)_{s}^{\dagger} .
$$

В силу известных результатов теории комплексных матриц каждый член суммы является положительным, и это справедливо для любой линейной комбинации таких членов с неотрицательными коэффициентами [11]. Наконец, поскольку вышеупомянутый изоморфизм сохраняет положительность [12], кватернионная эрмитова матрица (8) является положительной.

Прямое следствие предложения 1 состоит в том, что любое отображение (7) является положительным отображением в пространстве кватернионных матриц плотности. Более того, легко видеть, что любое отображение (8) может быть сведено к виду (7), если положить $V_{s}=\sqrt{\lambda_{s}} U_{s}$.

Заметим, что поскольку элементы матрицы $I_{n}$ вещественные, то на кватернионном векторном пространстве можно легко построить произведение Кронекера $\Phi \otimes I_{n}$, получив таким образом кватернионную положительную матрицу. Оказывается, что если матрица $\Phi$ вполне положительна (в смысле приведенного выше определения), 
то это гарантирует положительность матрицы $\Phi \otimes I_{n}$ в рамках кватернионного формализма; аналогичная ситуация имеет место и в комплексной квантовой механике.

Пусть $M(\mathbb{Q})$ и $M(\mathbb{C})$ - пространства кватернионных и комплексных $(n \times m)$-матриц, соответственно. Определим комплексную проекцию [4], [6] $P: M(\mathbb{Q}) \rightarrow M(\mathbb{C})$ посредством соотношения

$$
P[M]=\frac{1}{2}(M-i M i)=M_{\alpha},
$$

где $M=M_{\alpha}+j M_{\beta} \in M(\mathbb{Q})$. Тогда справедливо следующее предложение [6].

ПреДЛОЖЕНИЕ 2. Комплексная проекиия $\rho_{\alpha}$ любой кватернионной положительной эрмитовой матрицы $\rho=\rho_{\alpha}+j \rho_{\beta}$ является положительной эрмитовой матрицей.

Пусть теперь $\rho$ - кватернионная матрица плотности, а $U$ - некоторая кватернионная $(n \times n)$-матрица. Используя разложение на комплексную и чисто кватернионную части, $U=U_{\alpha}+U_{\beta} j$, получаем

$$
\begin{aligned}
U \rho U^{\dagger}= & U_{\alpha} \rho_{\alpha} U_{\alpha}^{\dagger}+U_{\beta} \rho_{\alpha}^{\mathrm{T}} U_{\beta}^{\dagger}-U_{\beta} \rho_{\beta} U_{\alpha}^{\dagger}+U_{\alpha} \rho_{\beta}^{*} U_{\beta}^{\dagger}+ \\
& +j\left(U_{\beta}^{*} \rho_{\alpha} U_{\alpha}^{\dagger}-U_{\alpha}^{*} \rho_{\alpha}^{\mathrm{T}} U_{\beta}^{\dagger}+U_{\alpha}^{*} \rho_{\beta} U_{\alpha}^{\dagger}+U_{\beta}^{*} \rho_{\beta}^{*} U_{\beta}^{\dagger}\right) .
\end{aligned}
$$

Отсюда в качестве прямого следствия предложения 2 получаем, что комплексное отображение

$$
\rho_{\alpha} \rightarrow U_{\alpha} \rho_{\alpha} U_{\alpha}^{\dagger}+U_{\beta} \rho_{\alpha}^{\mathrm{T}} U_{\beta}^{\dagger}-U_{\beta} \rho_{\beta} U_{\alpha}^{\dagger}+U_{\alpha} \rho_{\beta}^{*} U_{\beta}^{\dagger}
$$

является положительным отображением, и композиция двух любых таких отображений также является положительным отображением.

Результаты предложений 1 и 2 в совокупности доказывают следующее утверждение.

ПрЕДЛОЖЕНИЕ 3. Каждому вполне положителъному кватернионному отображению кватернионных матрии, плотности $\rho=\rho_{\alpha}+j \rho_{\beta}$ соответствует положительное комплексное отображение их комплексных проекиий $\rho_{\alpha}$.

Полагая $\rho_{\beta}=0$ в формуле (11), в качестве следствия предложения 3 снова получаем известный результат [4] (см. также [5]).

СлЕДСТВИЕ. Комплексная проекция вполне положительного кватернионного отображения комплексной матрицы плотности $\rho_{\alpha}$ есть комплексное разложимое отображение той же комплексной матрицы плотности $\rho_{\alpha}$. Справедливо также и обратное утверждение.

Заметим, наконец, что из линейности отображения $\Phi$ в пространстве кватернионных эрмитовых матриц $\rho$,

$$
\Phi\left(\rho_{1} a_{1}+\rho_{2} a_{2}\right)=\Phi\left(\rho_{1}\right) a_{1}+\Phi\left(\rho_{2}\right) a_{2}, \quad a_{1}, a_{2} \in \mathbb{R},
$$


очевидно следует линейность его комплексной проекции:

$$
\begin{aligned}
P\left[\Phi\left(\rho_{1} a_{1}+\rho_{2} a_{2}\right)\right] & =P\left[\Phi\left(\rho_{1}\right) a_{1}+\Phi\left(\rho_{2}\right) a_{2}\right]= \\
& =P\left[\Phi\left(\rho_{1}\right) a_{1}\right]+P\left[\Phi\left(\rho_{2}\right) a_{2}\right]=P\left[\Phi\left(\rho_{1}\right)\right] a_{1}+P\left[\Phi\left(\rho_{2}\right)\right] a_{2} .
\end{aligned}
$$

В заключение этого раздела сформулируем следующее предложение.

ПреДЛОЖЕНИЕ 4. Пусть $\rho_{\alpha}-n$-мерная комплексная положительная эрмитова матрица такая, что $\operatorname{rank} \rho_{\alpha}=m, 1<m \leqslant n$. Тогда существуют кососимметричные комплексные матрицы $\rho_{\beta}$ такие, что кватернионная эрмитова матрица $\rho=\rho_{\alpha}+j \rho_{\beta}$ является положительной, причем $m / 2 \leqslant \operatorname{rank} \rho \leqslant m$, если $m$ четное, $u(m+1) / 2 \leqslant \operatorname{rank} \rho \leqslant m$, если $m$ нечетное.

ДоКАЗАТЕЛЬСТВо. Предположим, что $m$ четное. Из эрмитовости матрицы $\rho_{\alpha}$

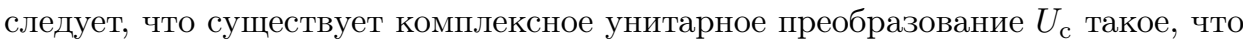

$$
U_{\mathrm{c}} \rho_{\alpha} U_{\mathrm{c}}^{\dagger}=\rho_{\alpha}^{\prime}=\operatorname{diag}\left(\lambda_{1}, \ldots, \lambda_{m}, 0, \ldots, 0\right),
$$

где $\lambda_{1}, \ldots, \lambda_{m}$ - положительные собственные значения матрицы $\rho_{\alpha}$.

Рассмотрим кватернионную матрицу

$$
\rho^{\prime}=\rho_{\alpha}^{\prime}+j \rho_{\beta}^{\prime}=\operatorname{diag}\left(\lambda_{1}, \ldots, \lambda_{m}, 0, \ldots, 0\right)+j \bigoplus_{r=1}^{n-\frac{m}{2}} B_{r},
$$

где $B_{r}=\left(\begin{array}{cc}0 & \mu_{r} \\ -\mu_{r} & 0\end{array}\right), \mu_{r} \in \mathbb{R}$, при $r=1, \ldots, m / 2$ и $B_{r}=0$ при $r=m / 2+1, \ldots, n-$ $m / 2$. Налагая ограничения $\lambda_{1} \lambda_{2} \geqslant \mu_{1}^{2}, \lambda_{3} \lambda_{4} \geqslant \mu_{2}^{2}, \ldots, \lambda_{m-1} \lambda_{m} \geqslant \mu_{m / 2}^{2}$, сразу получаем, что матрица $\rho^{\prime}$ является положительной и $m / 2 \leqslant \operatorname{rank} \rho \leqslant m$, где искомая кватернионная эрмитова матрица $\rho$ дается выражением

$$
\rho=U_{\mathrm{c}}^{\dagger} \rho^{\prime} U_{\mathrm{c}}=\rho_{\alpha}+j U_{\mathrm{c}}^{\mathrm{T}} \rho_{\beta}^{\prime} U_{\mathrm{c}} .
$$

Утверждение для случая нечетного $m$ может быть доказано с помощью замены $m / 2$ на $(m+1) / 2$ в определении $\rho_{\beta}^{\prime}$.

Предложение 4 дает возможность построить кватернионную матрицу плотности путем добавления к некоторой комплексной матрице плотности $\rho_{\alpha}$ подходящего слагаемого $j \rho_{\beta}$, сохраняя при этом положительность, а также, при необходимости, и ранг (для этого нужно выбирать $\rho_{\beta}$ так, чтобы rank $\rho=m$ ). Можно даже получить чистое состояние в том случае, когда $m=n=2$ и $\operatorname{rank} \rho=m / 2 ;$ примеры такого рода приведены в работах [5], [6] $]^{1)}$.

В более широком смысле предложение 4 оказывается полезным при классификации положительных комплексных отображений как комплексных проекций вполне положительных (и, в частности, унитарных) кватернионных отображений.

\footnotetext{
1)Напомним, что среднее значение любой чисто комплексной наблюдаемой в кватернионной квантовой механике не зависит от кватернионной части $j \rho_{\beta}$ состояния (см. раздел 2) и, таким образом, совпадает со средним значением, предсказываемым комплексной квантовой механикой.
} 


\section{4. КВАТЕРНИОННЫЕ УНИТАРНЫЕ ОТОБРАЖЕНИЯ И ИХ КОМПЛЕКСНЫЕ ПРОЕКЦИИ}

В этом разделе, в связи с важностью роли квантернионных унитарных отображений в квантовой механике, мы напомним некоторые результаты, касающиеся таких отображений, представляющих собой подмножество вполне положительных кватернионных отображений. В частности, ранее мы изучали комплексные проекции кватернионных унитарных отображений на инфинитезимальном уровне [6]. Мы напомним здесь некоторые результаты, чтобы сделать более понятной общую стратегию наших исследований.

В комплексной квантовой механике внутренняя динамика системы, взаимодействующей с внешней средой, не является, вообще говоря, унитарной, и во многих случаях может быть описана однопараметрической положительной полугруппой линейных отображений $\gamma_{t}=e^{t L}, t \geqslant 0$. Действие генератора $L$ на матрицу плотности смешанного состояния $\rho_{\alpha}$ задается основным уравнением Линдблада-Коссаковского $(\hbar=1)$

$$
\frac{d}{d t} \rho_{\alpha}=L\left[\rho_{\alpha}\right]=-\left[H_{\alpha}, \rho_{\alpha}(t)\right]+\sum_{r, s=1}^{n^{2}-1} C_{r s}\left(F_{r} \rho_{\alpha}(t) F_{s}^{\dagger}-\frac{1}{2}\left\{F_{r}^{\dagger} F_{s}, \rho_{\alpha}(t)\right\}\right),
$$

в которое входят следующие величины: антиэрмитов оператор Гамильтона $H_{\alpha}$, семейство $n^{2}-1$ квадратных матриц $F_{r}$ с нулевым следом, которые вместе с нормированной единицей $F_{0}=I_{n} / \sqrt{n}$ образуют ортонормированное множество (т.е. $\left.\operatorname{tr}\left(F_{r}^{\dagger} F_{s}\right)=\delta_{r s}\right)$, и эрмитова матрица $\left[C_{r s}\right][13]-[15]$. Кроме того, отображение $\gamma_{t}$ является вполне положительным тогда и только тогда, когда матрица $\left[C_{r s}\right]$ является положительной и $\operatorname{tr} H_{\alpha}=0$ [14].

В кватернионной квантовой механике уравнение Шредингера принимает вид

$$
\frac{d}{d t}|\psi\rangle=-H|\psi\rangle
$$

где $H=H_{\alpha}+j H_{\beta}-$ антиэрмитов оператор.

При рассмотрении зависящей от времени кватернионной унитарной динамики дифференциальное уравнение, описывающее временну́ю эволюцию состояния $\rho$, имеет вид

$$
\frac{d}{d t} \rho(t)=-[H(t), \rho(t)]
$$

и сводится к уравнению

$$
\frac{d}{d t} \rho_{\alpha}=-\left[H_{\alpha}, \rho_{\alpha}\right]+H_{\beta}^{*} \rho_{\beta}-\rho_{\beta}^{*} H_{\beta}
$$

для комплексной проекции матрицы плотности.

Член $H_{\beta}^{*} \rho_{\beta}-\rho_{\beta}^{*} H_{\beta}$ в уравнении (15), подобно негамильтонову члену в правой части уравнения (13), является эрмитовым, имеет нулевой след (вследствие циклического свойства следа) и образует генератор динамической полугруппы. В результате комплексная проекция любой кватернионной (зависящей от времени) унитарной 
динамики принадлежит множеству комплексных динамик, описываемых однопараметрической полугруппой положительных отображений [6].

Кроме того, напомним, что для плотного множества смешанных состояний максимального ранга была решена также и обратная задача, т.е. любая динамика комплексных матриц плотности максимального ранга, определяемая однопараметрической положительной полугруппой, может быть получена как комплексная проекция подходящей кватернионной унитарной динамики [6].

Рассмотрим, наконец, некоторые следствия предложений 1-4 применительно к кватернионным унитарным динамикам и соответствующим им комплексным проекциям на конечном уровне.

Пусть $\rho_{\alpha}-$ комплексная матрица плотности, а $\rho=\rho_{\alpha}+j \rho_{\beta}-$ кватернионная матрица плотности, полученная из $\rho_{\alpha}$ описанным в предложении 4 способом, так что $\operatorname{rank} \rho \leqslant \operatorname{rank} \rho_{\alpha}$. Тогда комплексная проекция унитарной кватернионной эволюции, действующей на $\rho$ по формуле

$$
\mathcal{U}: \rho \rightarrow U \rho U^{\dagger}=\rho_{\mathrm{c}},
$$

может во всех отношениях представлять комплексную неунитарную эволюцию матрицы $\rho_{\alpha}$ в комплексной матрице плотности $\rho_{\mathrm{c}}$ меньшего ранга.

\section{5. ПРИМЕР: $\mathbb{Q}-$ КУИТ}

Рассмотрим простой пример. Обозначим через $H_{\alpha}$ свободный комплексный антиэрмитов гамильтониан, описывающий частицу со спином $1 / 2$ в постоянном магнитном поле,

$$
H_{\alpha}=\frac{\omega}{2}\left(\begin{array}{cc}
i & 0 \\
0 & -i
\end{array}\right),
$$

а через $j H_{\beta}$ обозначим антиэрмитов чисто кватернионный постоянный потенциал,

$$
j H_{\beta}=\left(\begin{array}{cc}
0 & j v \\
j v & 0
\end{array}\right), \quad v>0 .
$$

Собственные значения и соответствующие (ортонормированные) собственные векторы кватернионного гамильтониана $H=H_{\alpha}+j H_{\beta}$ даются формулами [16]

$$
i E_{ \pm}=i\left(\frac{\omega}{2} \pm v\right), \quad\left|\psi_{ \pm}\right\rangle=\left(\begin{array}{c} 
\pm i \\
j
\end{array}\right) \frac{1}{\sqrt{2}} .
$$

Унитарный эволюционный оператор имеет вид

$$
\begin{aligned}
U(t) & =e^{-H t}=\left|\psi_{+}\right\rangle e^{-i E_{+} t}\left\langle\psi_{+}|+| \psi_{-}\right\rangle e^{-i E_{-} t}\left\langle\psi_{-}\right|= \\
& =\frac{1}{2}\left(\begin{array}{cc}
e^{-i E_{+} t}+e^{-i E_{-} t} & \left(e^{-i E_{-} t}-e^{-i E_{+} t}\right) k \\
\left(e^{i E_{+} t}-e^{i E_{-} t}\right) k & e^{i E_{+} t}+e^{i E_{-} t}
\end{array}\right)=U_{\alpha}+U_{\beta} j .
\end{aligned}
$$

Если вспомнить, что комплексное разложимое отображение, соответствующее транспозиции в двумерном комплексном гильбертовом пространстве, может быть записано в виде

$$
\rho_{\alpha} \rightarrow \rho_{\alpha}^{\mathrm{T}}=\frac{1}{2}\left(\sigma_{0} \rho_{\alpha} \sigma_{0}+\sigma_{1} \rho_{\alpha} \sigma_{1}-\sigma_{2} \rho_{\alpha} \sigma_{2}+\sigma_{3} \rho_{\alpha} \sigma_{3}\right),
$$


где

$$
\sigma_{0}=\left(\begin{array}{ll}
1 & 0 \\
0 & 1
\end{array}\right), \quad \sigma_{1}=\left(\begin{array}{cc}
0 & 1 \\
1 & 0
\end{array}\right), \quad \sigma_{2}=\left(\begin{array}{cc}
0 & -i \\
i & 0
\end{array}\right), \quad \sigma_{3}=\left(\begin{array}{cc}
1 & 0 \\
0 & -1
\end{array}\right)
$$

то вид Крауса-Стайнспринга комплексной проекции кватернионного унитарного отображения (20) на подмножество комплексных матриц плотности $\rho_{0 \alpha}=\rho_{\alpha}(0)$ дается выражением

$$
U_{\alpha} \rho_{0 \alpha} U_{\alpha}^{\dagger}+U_{\beta} \rho_{0 \alpha} U_{\beta}^{\dagger}+U_{\beta} \sigma_{1} \rho_{0 \alpha} \sigma_{1} U_{\beta}^{\dagger}+U_{\beta} \sigma_{3} \rho_{0 \alpha} \sigma_{3} U_{\beta}^{\dagger}-U_{\beta} \sigma_{2} \rho_{0 \alpha} \sigma_{2} U_{\beta}^{\dagger}
$$

(заметим, что из-за знака минус в последнем члене это отображение является положительным, но не вполне положительным), в то время как вид комплексной проекции кватернионного унитарного отображения (20) на подмножество чисто кватернионных матриц плотности $\rho_{0 \alpha}+j \rho_{0 \beta}$ получается путем добавления члена $-U_{\beta} \rho_{0 \beta} U_{\alpha}^{\dagger}+$ $U_{\alpha} \rho_{0 \beta}^{*} U_{\beta}^{\dagger}$ к последнему выражению:

$$
\begin{gathered}
U_{\alpha} \rho_{0 \alpha} U_{\alpha}^{\dagger}+U_{\beta} \rho_{0 \alpha} U_{\beta}^{\dagger}+U_{\beta} \sigma_{1} \rho_{0 \alpha} \sigma_{1} U_{\beta}^{\dagger}-U_{\beta} \sigma_{2} \rho_{0 \alpha} \sigma_{2} U_{\beta}^{\dagger}+ \\
+U_{\beta} \sigma_{3} \rho_{0 \alpha} \sigma_{3} U_{\beta}^{\dagger}-U_{\beta} \rho_{0 \beta} U_{\alpha}^{\dagger}+U_{\alpha} \rho_{0 \beta}^{*} U_{\beta}^{\dagger} .
\end{gathered}
$$

Рассмотрим комплексное начальное состояние

$$
\rho(0)=\left(\begin{array}{cc}
a & 0 \\
0 & 1-a
\end{array}\right)=\frac{1}{2}\left(\sigma_{0}-\sigma_{3}\right)+a \sigma_{3}, \quad a \in \mathbb{R}, \quad 0 \leqslant a \leqslant 1 .
$$

Тогда из выражения (20) имеем

$$
\rho(t)=U(t) \rho(0) U^{\dagger}(t)=\frac{1}{2}\left[\sigma_{0}+\sigma_{3}(2 a-1) \cos (2 v t)-k \sigma_{2}(2 a-1) \sin (2 v t)\right] .
$$

Заметим, что $S(\rho(0))=-a \ln a-(1-a) \ln (1-a)=S(\rho(t))$.

Комплексная проекция $\rho_{\alpha}(t)$ величины $\rho(t)=\rho_{\alpha}(t)+j \rho_{\beta}(t)$ имеет вид

$$
\rho_{\alpha}(t)=\frac{1}{2}\left[\sigma_{0}+\sigma_{3}(2 a-1) \cos (2 v t)\right]
$$

и, очевидно, является положительной; более того, из выражения (6) получаем

$$
\begin{aligned}
S\left(\rho_{\alpha}(t)\right)=- & \frac{1}{2}[(1+(2 a-1) \cos (2 v t)) \ln (1+(2 a-1) \cos (2 v t))+ \\
& +(1-(2 a-1) \cos (2 v t)) \ln (1-(2 a-1) \cos (2 v t))] .
\end{aligned}
$$

Рассмотрим спиновую наблюдаемую, которая в кватернионной квантовой механике связана с триплетом комплексных операторов. Среднее значение $z$-компоненты $s_{z}=\sigma_{3} / 2$ в случае, когда система находится в кватернионном смешанном состоянии (25), дается формулой

$$
\left\langle s_{z}\right\rangle=\operatorname{Re} \operatorname{tr}\left(s_{z} \rho(t)\right)=\frac{1}{2}(2 a-1) \cos (2 v t),
$$


и в соответствии с (5)

$$
\operatorname{Re} \operatorname{tr}\left(s_{z} \rho(t)\right)=\operatorname{tr}\left(s_{z} \rho_{\alpha}(t)\right) .
$$

Простое вычисление показывает, что (положительно-определенная) наблюдаемая $|H|$ (энергия) имеет вид [7]

$$
|H|=\left|\psi_{+}\right\rangle E_{+}\left\langle\psi_{+}|+| \psi_{-}\right\rangle E_{-}\left\langle\psi_{-}\right|=\frac{1}{2}\left(\sigma_{0} \omega+k \sigma_{2} 2 v\right)
$$

и ее среднее значение дается формулой

$$
\langle|H|\rangle=\operatorname{Retr}(|H| \rho(t))=\frac{\omega}{2}=\operatorname{Re} \operatorname{tr}(|H| \rho(0)) .
$$

Теперь генератор однопараметрической полугруппы, связанной с комплексной проекцией кватернионной унитарной динамики, которая задается формулой (25), может быть вычислен непосредственно:

$$
L\left[\rho_{\alpha}(t)\right]=-\left[H_{\alpha}, \rho_{\alpha}\right]+H_{\beta}^{*} \rho_{\beta}-\rho_{\beta}^{*} H_{\beta}=\sigma_{3}(1-2 a) v \sin (2 v t) .
$$

Рассмотрим теперь чистое кватернионное начальное состояние

$$
\rho(0)=\rho_{\alpha}(0)+j \rho_{\beta}(0)=\frac{1}{2}\left(\sigma_{0}-\sigma_{3}\right)+a \sigma_{3}-k \sigma_{2} \frac{\sqrt{1-(2 a-1)^{2}}}{2},
$$

где к матрице плотности (24) добавлен кватернионный член $j \rho_{\beta}(0)$ (полученный по рецепту предложения 4) так, чтобы матрица $\rho(0)$ по-прежнему оставалась положительной и имела ранг $\operatorname{rank} \rho(0)=1$.

Тогда из выражения (20) получаем эволюцию

$$
\begin{aligned}
\rho(t) & =U(t) \rho(0) U^{\dagger}(t)= \\
& =\frac{1}{2}\left[\sigma_{0}+\sigma_{3}\left((2 a-1) \cos (2 v t)-\sqrt{1-(2 a-1)^{2}} \sin (2 v t)\right)-k \sigma_{2}(2 a-1) \sin (2 v t)\right],
\end{aligned}
$$

комплексная проекция которой дается формулой

$$
\rho_{\alpha}(t)=\frac{1}{2}\left[\sigma_{0}+\sigma_{3}\left((2 a-1) \cos (2 v t)-\sqrt{1-(2 a-1)^{2}} \sin (2 v t)\right)\right] .
$$

Среднее значение $z$-компоненты спиновой наблюдаемой теперь равно

$$
\left\langle s_{z}\right\rangle=\operatorname{Retr}\left(s_{z} \rho_{\alpha}(t)\right)=\frac{1}{2}\left[(2 a-1) \cos (2 v t)-\sqrt{1-(2 a-1)^{2}} \sin (2 v t)\right],
$$

а среднее значение энергии равно $\omega / 2$.

Наконец, генератор однопараметрической полугруппы, связанной с комплексной проекцией кватернионной унитарной динамики для матрицы плотности, которая задается формулой (31), может быть вычислен непосредственно:

$$
L\left[\rho_{\alpha}(t)\right]=-\left[H_{\alpha}, \rho_{\alpha}\right]+H_{\beta}^{*} \rho_{\beta}-\rho_{\beta}^{*} H_{\beta}=-\sigma_{3} v[2 \sqrt{a(1-a)} \cos (2 v t)+(2 a-1) \sin (2 v t)] .
$$

Этот простой пример может иметь некоторые интересные физические приложения, поскольку постоянный кватернионный потенциал сильно влияет на величину спина, в то же время оставляя энергию системы без изменений.

2 Теоретическая и математическая физика, т. 151, № 3, 2007 г. 


\section{6. ЗАКЛЮЧЕНИЕ}

В разделе 3 было показано, что комплексные проекции вполне положительных кватернионных отображений (и, в частности, унитарных кватернионных отображе-

ний) являются положительными комплексными отображениями, которые в общем случае оказываются не вполне положительными. С другой стороны, любое положительное комплексное отображение, полученное как комплексная проекция унитарной кватернионной динамики, принадлежит множеству комплексных динамик, порождаемых действием однопараметрической полугруппы. Таким образом, мы имеем систематический метод для построения комплексных динамик, управляемых положительными (но, вообще говоря, не вполне положительными) однопараметрическими полугруппами, путем взятия комплексных проекций кватернионных унитарных динамик. Напомним, кроме того, что условия на матрицу Коссаковского генератора однопараметрической полугруппы (13), необходимые и достаточные для того, чтобы соответствующее динамическое отображение было положительным, но не вполне положительным, до сих пор не найдены. Мы полагаем, что дальнейшие более глубокие исследования комплексных проекций кватернионных отображений могут привести к решению этой задачи.

Благодарности. Г. Сколаричи выражает благодарность Г. Мармо и А. Коссаковскому за полезные обсуждения. Эта работа была частично поддержана СІСҮТ (грант FPA2004-02948), DGIID-DGA (грант 2005-E24/2) и PRIN SINTESI.

\section{Список литературы}

[1] S. Weinberg, Phys. Rev. Lett., 62 (1989), 485.

[2] M. Asorey, A. Kossakowski, G. Marmo, E. C. G. Sudarshan, Open Syst. Inf. Dyn., 12 (2005), 319.

[3] V.I. Man'ko, G. Marmo, E. C. G. Sudarshan, F. Zaccaria, Phys. Lett. A, 327 (2004), 353.

[4] A. Kossakowski, Rep. Math. Phys., 46 (2000), 393.

[5] G. Scolarici, L. Solombrino, "Complex entanglement and quaternionic separability", The Foundations of Quantum Mechanics. Historical Analysis and Open Questions (Cesena, Italy, 2004), eds. C. Garola, A. Rossi, S. Sozzo, World Sci., Singapore, 2006, 301.

[6] M. Asorey, G. Scolarici, J. Phys. A, 39 (2006), 9727.

[7] S. L. Adler, Quaternionic Quantum Mechanics and Quantum Fields, Internat. Ser. Monogr. Phys., 88, Oxford Univ. Press, New York, 1995.

[8] D. Finkelstein, J. M. Jauch, S. Sciminovich, D. Speiser, J. Math. Phys., 4 (1963), 788.

[9] K. Kraus, States, Effects and Operations. Fundamental Notions of Quantum Theory, Lect. Notes Phys., 190, eds. A. Böhm, J. D. Dollard, W. H. Wootters, Springer, Berlin, 1983.

[10] W. F. Stinespring, Proc. Amer. Math. Soc., 6 (1955), 211.

[11] R. A. Horn, C. R. Johnson, Matrix Analysis, v. 1, Cambridge Univ. Press, Cambridge, 1985.

[12] F. Zhang, Linear Algebra Appl., 251 (1997), 21.

[13] A. Kossakowski, Rep. Math. Phys., 43 (1972), 247.

[14] V. Gorini, A. Kossakowski, E. C. G. Sudarshan, J. Math. Phys., 17 (1976), 821.

[15] G. Lindblad, Comm. Math. Phys., 48 (1976), 119.

[16] S. De Leo, G. Scolarici, L. Solombrino, J. Math. Phys., 43 (2002), 5815. 